\section{JOURNAL OF \\ Veterinary Emergency \\ and Critical Care}

\title{
Comparison of 4 point-of-care blood gas analyzers for arterial blood gas analysis in healthy dogs and dogs with cardiopulmonary disease
}

\author{
Elodie Roels, DVM; Kris Gommeren, DVM, DECVIM; Frédéric Farnir, Ir, PhD; \\ François Delvaux, MS; Frédéric Billen, DVM, PhD, DECVIM and Cécile Clercx, DVM, PhD, DECVIM
}

\begin{abstract}
Objective - To compare blood gas results obtained from 4 point-of-care (POC) blood gas analyzers under routine working conditions in order to determine their interchangeability.

Design - Prospective study.

Setting - University teaching hospital.

Animals - Arterial blood samples from 34 dogs, 22 presented for cardiorespiratory disease and 12 healthy experimental Beagles.

Measurements - Each sample was analyzed by 4 POC blood gas analyzers in a random order: Cobas b-123 POC system, IRMA TruPoint, Idexx VetStat, and ABL80 FLEX. Values obtained for $\mathrm{pH}$, partial pressure of oxygen $\left(\mathrm{PO}_{2}\right)$, and partial pressure of carbon dioxide $\left(\mathrm{PCO}_{2}\right)$ were compared between analyzers using a statistical mixed linear model and the Bland-Altman method; $P<0.05$ was set as the level of significance. One sample was also repeatedly measured 5 times on each machine to calculate and compare intra-analyzer variance for each measured variable using Fisher-statistics.

Results $-\mathrm{PO}_{2}$ and $\mathrm{PCO}_{2}$ values were significantly higher when measured with the Idexx and IRMA machine than with Cobas and ABL80 $\left(\mathrm{PO}_{2}: P<0.001 ; \mathrm{PCO}_{2}: P<0.05\right)$. $\mathrm{pH}$ values were significantly higher when measured with the Idexx instrument than with others' devices $(P<0.01)$. An interaction between health status and results delivered by devices was found only for $\mathrm{PO}_{2}$ values. There was no significant difference in intraanalyzer variance between the 4 machines, for any of the measured variables.

Conclusions $-\mathrm{PO}_{2}, \mathrm{PCO}_{2}$, and $\mathrm{pH}$ differed significantly between the 4 analyzers. Differences in $\mathrm{PO}_{2}$ results were substantial and clinically relevant. All 4 analyzers displayed good intra-analyzer variance. Consequently, serial blood gas analysis should be performed on a single device and interpreted in accordance of the device's specific reference intervals.
\end{abstract}

(J Vet Emerg Crit Care 2016; 00(0): 1-8) doi: 10.1111/vec.12469

Keywords: acid-base monitoring, canine, laboratory equipment, oxygenation indices

From the Department of Clinical Sciences (Roels, Gommeren, Delvaux, Billen, Clercx) and the Department of Animal Productions, Biostatistics and Bioinformatics applied to Veterinary Sciences (Farnir), Faculty of Veterinary Medicine, University of Liege, Liège, Belgium.

Elodie Roels receives a grant from the Belgian Foundation of Scientific Research, FRS-FNRS.

The authors declare no conflicts of interest.

Presented in part at the 23rd Congress of the European College of Veterinary Internal Medicine - Companion Animals (ECVIM-CA), Liverpool, UK, September 2013 and at the 3rd FMV Scientific Day, Liège, Belgium, October 2013.

Address correspondence and reprint requests to

Dr. Elodie Roels, Department of Clinical Sciences, Faculty of Veterinary

Medicine, Bd de Colonster, 20 (B44), 4000 Liège, Belgium.

Email: eroels@ulg.ac.be

Submitted February 24, 2014; Accepted April 11, 2014.

\section{Abbreviations}

lsm Least square mean

$\mathrm{PCO}_{2}$ partial pressure of carbon dioxide

$\mathrm{PO}_{2} \quad$ partial pressure of oxygen

POC point-of-care

\section{Introduction}

Arterial blood gas analysis is frequently used in critical care and research to assess the efficacy of respiration, tissue oxygenation, and acid-base balance. ${ }^{1}$ Arterial blood gases play an important role in the 
Table 1: Precision analysis: coefficient of variation (\%) obtained from the same sample run 5 times on each device

\begin{tabular}{lcccc}
\hline Parameter & Cobas & Idexx & IRMA & ABL80 \\
\hline $\mathrm{PO}_{2}$ & 2.08 & 1.75 & 3.50 & 4.72 \\
$\mathrm{PCO}_{2}$ & 0.91 & 1.38 & 1.43 & 8.33 \\
$\mathrm{pH}$ & 0.02 & 0.07 & 0.24 & 0.39 \\
\hline
\end{tabular}

$\mathrm{PCO}_{2}$, partial pressure of carbon dioxide; $\mathrm{PO}_{2}$, partial pressure of oxygen.

monitoring of patients receiving oxygen supplementation and in the work-up and management of critically ill patients with cardiopulmonary impairment. Assessment of blood gases may help to obtain a diagnosis, characterize disease severity, and allow monitoring of disease progression and response to treatment. ${ }^{2-6}$ Recent technological advances have allowed the production and marketing of point-of-care (POC) devices allowing rapid analysis of important variables. However, little is known about how results compare between different analyzers, even though many large centers work with different POC devices in their routine and emergency laboratories. Therefore, it is important to know if a systematic difference in absolute values or a difference in precision exists, related to the type of analyzer used, before making a clinical decision. Accordingly, the primary objective of this study was to compare blood gas values obtained from 4 POC blood gas analyzers under routine working conditions for samples from healthy dogs and dogs presented for cardiopulmonary impairment, breathing room air, and highlight possible clinically significant differences between the results obtained by different instruments. A second objective was to determine the ease-of-use in a clinical setting of each device.

\section{Materials and Methods}

The protocol for this study was approved by the University of Liege's ethics committee. Informed owner consent was obtained where applicable. One single operator performed all blood sampling and blood gas measurements to avoid variation due to operator effects.

\section{Patient samples}

Arterial blood samples were prospectively collected during a 4-month period, from dogs breathing room air. Animals used were either experimental healthy Beagles or dogs presented for cardiopulmonary clinical evaluation. Arterial blood was drawn from the metatarsal artery following placement of a 22-Ga intra-arterial catheter in 1 experimental Beagle for precision estimation. In all other dogs, a 25-Ga needle and $2 \mathrm{~mL}$ heparinized syringe $^{a}$ were used after subcutaneous injection of local anesthetic $^{\mathrm{b}}$ for analgesia. Immediately after blood sam- pling, air bubbles were removed from the syringe and the syringe closed with an airtight lid before being gently mixed by repeated inversion. Blood gas analysis was then performed immediately. The patient's rectal temperature was recorded to permit correction of measurements according to body temperature.

\section{Devices}

Four POC blood gas analyzers were evaluated: Cobas, ${ }^{\mathrm{C}}$ IRMA, ${ }^{\mathrm{d}}$ Idexx, ${ }^{\mathrm{e}}$ and ABL80. ${ }^{\mathrm{f}}$ All instruments were calibrated, maintained, and operated according to their respective manufacturer's instructions. Machines were installed adjacent to each other in order to minimize the time interval between measurements. The evaluated analytes were $\mathrm{pH}$, partial pressure of oxygen $\left(\mathrm{PO}_{2}\right)$, and partial pressure of carbon dioxide $\left(\mathrm{PCO}_{2}\right)$. In case of assay failure, it was impossible to repeat the analysis due to the limited quantity of sampled blood.

\section{Precision}

To estimate and compare precision, a single sample was drawn into four $2 \mathrm{~mL}$ heparinized syringes ${ }^{\mathrm{a}}$ through an intra-arterial catheter in 1 experimental healthy Beagle. The sample was analyzed 5 times on each machine; intraanalyzer variance was calculated for each parameter.

\section{Comparison}

Each blood sample obtained from the experimental healthy Beagles and client-owned dogs was analyzed on the 4 instruments in a random order. All analyses were performed within 15 minutes after sampling. After each analysis, air bubbles, introduced into the syringe during sample aspiration, were removed; the sample was closed with the airtight cap and thoroughly remixed by hand prior to analysis on the following analyzer.

\section{Statistics}

POC analyzer results were compared using a statistical mixed linear model ${ }^{g}$ integrating interaction between health status and results delivered by each device. Interaction was included in the model in order to investigate if differences observed between devices could vary according to the range of values measured. Values are given as least square mean $(\mathrm{lsm}) \pm \mathrm{SE}$. Statistical significance was set at $P<0.05$. Measurements obtained from the 4 devices were also compared using the Bland-Altman method. ${ }^{\text {h }}$ Results are expressed as bias (the mean difference) \pm SE ( $95 \%$ confidence interval). Intra-analyzer variances measured for each variable and for each device were compared using Fisher statistics ${ }^{\mathrm{h}} ; P<0.05$ was chosen as the level of significance. Failure rates of 
Table 2: Results of blood gas analysis

\begin{tabular}{lcccc}
\hline Parameter & Cobas & ABL80 & IRMA & Idexx \\
\hline$n$ & 33 & 28 & 28 & 31 \\
$\mathrm{PO}_{2}(\mathrm{~mm} \mathrm{Hg})$ & $78.4 \pm 4.0$ & $79.6 \pm 4.0$ & $88.2 \pm 4.0^{*}$ & $88.6 \pm 4.0^{*}$ \\
& $(42.6-106.6)$ & $(31.0-106.0)$ & $(31.7-119.0)$ & $(48.0-113.0)$ \\
$\mathrm{PCO}_{2}(\mathrm{~mm} \mathrm{Hg})$ & $37.1 \pm 0.8$ & $38.0 \pm 0.8$ & $40.2 \pm 0.8^{*}$ & $(33.6-50.3)$ \\
& $(27.7-47.2)$ & $(30.0-50.0)$ & $(34.0-52.0)$ \\
$\mathrm{pH}$ & $7.369 \pm 0.009$ & $7.370 \pm 0.009$ & $7.369 \pm 0.009$ & $7.388 \pm 0.009^{\dagger}$ \\
& $(7.242-7.446)$ & $(7.200-7.420)$ & $(7.212-7.444)$ & $(7.220-7.450)$ \\
\hline
\end{tabular}

Data are expressed as least square mean $\pm \mathrm{SE}$ (range).

* Indicates statistical difference $(P<0.05)$ between Cobas and ABL80.

${ }^{\dagger}$ Indicates statistical difference $(P<0.001)$ between Cobas, ABL80 and IRMA.

Table 3: Results of the statistical mixed linear model and Bland-Altman analysis

\begin{tabular}{|c|c|c|c|c|c|c|c|}
\hline \multicolumn{2}{|c|}{ Statistics parameters } & \multirow{2}{*}{$\begin{array}{c}\begin{array}{c}\text { Cobas } \\
\text { IRMA }\end{array} \\
P<0.001\end{array}$} & \multirow{2}{*}{$\begin{array}{c}\begin{array}{c}\text { Cobas } \\
\text { Idexx }\end{array} \\
P<0.001\end{array}$} & \multirow{2}{*}{$\begin{array}{c}\text { Cobas } \\
\text { ABL80 } \\
\text { NS }\end{array}$} & \multirow{2}{*}{$\begin{array}{c}\text { Idexx } \\
\text { IRMA }\end{array}$} & \multirow{2}{*}{$\begin{array}{c}\text { IRMA } \\
\text { ABL80 } \\
P<0.001\end{array}$} & \multirow{2}{*}{$\begin{array}{c}\begin{array}{c}\text { Idexx } \\
\text { ABL80 }\end{array} \\
P<0.001\end{array}$} \\
\hline Statistical mixed & $\mathrm{PO}_{2}$ & & & & & & \\
\hline & $\mathrm{PCO}_{2}$ & $P=0.0061$ & $P=0.0005$ & NS & NS & $P=0.0548$ & $P=0.0089$ \\
\hline & $\mathrm{pH}$ & NS & $P<0.001$ & NS & $P<0.001$ & NS & $P<0.001$ \\
\hline \multirow[t]{3}{*}{$\begin{array}{l}\text { Bland-Altman } \\
\text { analysis }\end{array}$} & $\begin{array}{l}\mathrm{PO}_{2} \\
(\mathrm{~mm} \mathrm{Hq})\end{array}$ & $\begin{array}{l}9.5 \pm 7.5 \\
(-5.3-24.3)\end{array}$ & $\begin{array}{l}10.5 \pm 6.0 \\
(-1.3-22.3)\end{array}$ & $\begin{array}{l}1.8 \pm 4.3 \\
(-6.6-10.3)\end{array}$ & $\begin{array}{c}0.4 \pm 6.4 \\
(-12.1-12.9)\end{array}$ & $\begin{array}{l}-7.2 \pm 6.4 \\
(-19.7-5.3)\end{array}$ & $\begin{array}{l}-7.9 \pm 4.1 \\
(-16.0-0.2)\end{array}$ \\
\hline & $\begin{array}{l}\mathrm{PCO}_{2} \\
(\mathrm{~mm} \mathrm{Hg})\end{array}$ & $\begin{array}{l}2.8 \pm 1.9 \\
(-0.9-6.6)\end{array}$ & $\begin{array}{l}3.8 \pm 1.7 \\
(0.5-7.2)\end{array}$ & $\begin{array}{l}0.5 \pm 2.6 \\
(-4.7-5.8)\end{array}$ & $\begin{array}{l}-0.8 \pm 1.9 \\
(-4.4-2.9)\end{array}$ & $\begin{array}{l}-2.3 \pm 2.6 \\
(-7.3-2.7)\end{array}$ & $\begin{array}{l}-3.3 \pm 2.8 \\
(-8.8-2.2)\end{array}$ \\
\hline & $\mathrm{pH}$ & $\begin{array}{l}-0.001 \pm 0.020 \\
(-0.041-0.039)\end{array}$ & $\begin{array}{l}0.019 \pm 0.018 \\
(-0.016-0.054)\end{array}$ & $\begin{array}{c}-0.0004 \pm 0.025 \\
(-0.049-0.048)\end{array}$ & $\begin{array}{l}-0.020 \pm 0.023 \\
(-0.065-0.025)\end{array}$ & $\begin{array}{l}-0.001 \pm 0.031 \\
(-0.062-0.060)\end{array}$ & $\begin{array}{l}-0.019 \pm 0.024 \\
(-0.066-0.028)\end{array}$ \\
\hline
\end{tabular}

For Bland-Altman analysis, data are expressed as bias \pm SE (95\% confidence interval).

NS indicates not significant $(P>0.05)$.

$\mathrm{PCO}_{2}$, partial pressure of carbon dioxide; $\mathrm{PO}_{2}$, partial pressure of oxygen.

the devices were compared with a chi-square test ${ }^{\text {h }}$ with a threshold at $5 \%$.

\section{Results}

\section{Patient samples}

Thirty-four dogs, 18 male and 16 female, aged from 9 months to 15 years (median age, 6.8 years) and weighing $2.3-43 \mathrm{~kg}$ (median weight, $12.4 \mathrm{~kg}$ ) were included in this study. Twelve healthy experimental Beagle dogs, as assessed by history and a complete physical examination, were included. Twenty-two dogs of various breeds (4 Shih-Tzus, 3 Bulldogs, 2 Beagles, 2 Yorkshire Terriers, 2 mixed breed dogs, and 1 each of: Pug, Jack Russell Terrier, Boxer, Scottish Terrier, Labrador, King Charles Spaniel, Bernese Mountain Dog, Basset Hound, and Border Collie), presented for various cardiopulmonary diseases, were also included. Clinical diagnosis at the time of arterial blood sampling was dyspnea of unknown origin in 6 dogs, chronic cough of unknown origin in 4 dogs, brachycephalic airway obstruction syndrome in 4 dogs, bronchopneumonia in 2 dogs, lung neoplasia in 2 dogs, reversed patent ductus arteriosus in $1 \mathrm{dog}$, methemoglobinemia in $1 \mathrm{dog}$, laryngeal paralysis in 1 $\mathrm{dog}$, and eosinophilic bronchopneumopathy in $1 \mathrm{dog}$.

\section{Devices}

In 4 cases, analysis with the IRMA ( 3 out of 4 ) or the Idexx ( 1 out of 4 ) machine was technically impossible due to unavailability of cartridges at the time of testing. Failure rate was 6 of $34(18 \%)$ for the ABL80, 3 of $31(10 \%)$ for the IRMA, 2 of $33(6 \%)$ for the Idexx, and 1 of $34(3 \%)$ for the Cobas machine. Failure was due to sample mishandling in 3 out of 6 ABL80 failed samples and 1 out of 2 Idexx failed samples. Clot formation occurred in 1 of 2 Idexx failed samples, while all other failed samples on all devices were due to air bubble aspiration (ABL80 3 out of 6; IRMA 3 out of 3; Cobas 1 out of 1). The difference between the failure proportion obtained for each device was not statistically significant $(P=0.176)$.

\section{Precision}

No significant difference in intra-analyzer variance was found for the 4 analyzers for any of the variables 
Table 4: Partial pressure of oxygen values obtained in patients presented for cardiopulmonary disease with clinically discordant results between devices

\begin{tabular}{lcccc}
\hline $\mathbf{P O}_{\mathbf{2}}(\mathbf{m m ~ H g})$ & Cobas & Idexx & ABL80 & IRMA \\
\hline Patient 1 & 80.9 & 94 & 87 & 93.9 \\
Patient 2 & 80.3 & 96 & - & - \\
Patient 3 & 74.3 & 100 & - & - \\
\hline
\end{tabular}

Hypoxemia was defined as partial pressure of oxygen $\left(\mathrm{PO}_{2}\right)<90 \mathrm{~mm} \mathrm{Hg}$.

studied $(P>0.499)$. However, the ABL80 device demonstrated a coefficient of variation $>4 \%$ for both $\mathrm{PO}_{2}$ and $\mathrm{PCO}_{2}$ values while the other devices demonstrated a coefficient of variation $\leq 3.5 \%$ for the same 2 analytes (Table 1).

\section{Comparison}

$\mathrm{PO}_{2}, \mathrm{PCO}_{2}$, and $\mathrm{pH}$ results for all 4 devices are presented in Table 2. $\mathrm{PO}_{2}$ differed significantly $(P<0.001)$ between devices, with significantly higher mean values on the Idexx and IRMA devices compared to the Cobas and ABL80 machines (Table 3). This difference was also reflected by larger bias observed between Cobas and Idexx, Cobas and IRMA, ABL80 and Idexx, and ABL80 and IRMA (Table 3). Bland-Altman plots for $\mathrm{PO}_{2}$ results are presented in Figure 1. Normal reference values for $\mathrm{PO}_{2}$ range from 90 to $100 \mathrm{~mm} \mathrm{Hg.}{ }^{1}$ When defining hypoxemia as a $\mathrm{PO}_{2}$ lower than $90 \mathrm{~mm} \mathrm{Hg}$, clinical discording results were found in 3 patients presented for cardiopulmonary disease (Table 4). For $\mathrm{PCO}_{2}$, mean values were significantly $(P<0.05)$ higher on the Idexx and IRMA instruments compared to the Cobas and ABL80 devices (Table 3). Bland-Altman plots for $\mathrm{PCO}_{2}$ results are presented in Figure 2. $\mathrm{pH}$ values obtained with the Idexx machine were significantly $(P<0.001)$ higher than those measured with Cobas, IRMA, and ABL80 devices (Table 3). Bland-Altman plots for $\mathrm{pH}$ results are presented in Figure 3. For $\mathrm{pH}$ and $\mathrm{PCO}_{2}$ variables, no interaction between the results obtained from each analyzer and the health status of the dog was found. For $\mathrm{PO}_{2}$, a significant $(P=0.008)$ interaction between values measured from each machine and health status was observed. In dogs presented for cardiopulmonary disease, the Idexx device had the higher $\mathrm{PO}_{2} \mathrm{lsm}(78.4 \pm$ $4.7 \mathrm{~mm} \mathrm{Hg}$ ) compared to IRMA (73.8 \pm 4.8$)$, ABL80 (68.4 $\pm 4.8)$ and Cobas $(66.7 \pm 4.7)$ machines. At higher $\mathrm{PO}_{2}$ values the difference between the IRMA device and other devices tended to be larger (Figure 1), which might explain why in healthy experimental dogs the IRMA machine displayed higher $\mathrm{PO}_{2} \mathrm{lsm}(102.6 \pm 6.4)$ compared to Idexx (98.8 \pm 6.4$), \mathrm{ABL} 80(90.9 \pm 6.4)$, and Cobas (90.1 $\pm 6.4)$.

\section{Discussion}

In the veterinary literature, limited data have been published concerning the performance of blood gas analyzers. To the authors' knowledge, this is the first report comparing 4 different POC machines for arterial blood gas analysis in dogs. The aim of this study was to compare results obtained from 4 different analyzers and to determine whether they are interchangeable without a risk of clinically relevant discrepancies. Aliquots of arterial blood samples from 34 dogs were analyzed on all 4 devices for $\mathrm{pH}, \mathrm{PO}_{2}$, and $\mathrm{PCO}_{2}$. We found that $\mathrm{PO}_{2}$ measurements differed significantly $(P<0.001)$ between devices and that difference in $\mathrm{PO}_{2}$ values obtained from different machines could reach $20 \mathrm{~mm} \mathrm{Hg}$ in some cases. A significant interaction was observed between health status and $\mathrm{PO}_{2}$ results delivered by each analyzer. The Idexx device recorded the highest lsm values in dogs presented for cardiopulmonary disease while the IRMA device displayed higher $\mathrm{PO}_{2}$ results in the healthy dogs. These findings illustrate the potential for different clinical interpretations and indicate that values obtained from different POC machines must be interpreted cautiously when used for diagnostic and patient monitoring. For example, in our study, 3 patients presented for cardiopulmonary disease, had $\mathrm{PO}_{2}$ values above $90 \mathrm{~mm} \mathrm{Hg}$ on the Idexx device (and IRMA for patient number 1), yet Cobas (and ABL80 for patient number 1) reported values lower than $90 \mathrm{~mm} \mathrm{Hg}$, implying hypoxemia. In these instances a clinical decision was made to supplement these patients with oxygen. For $\mathrm{PCO}_{2}$, statistically significant $(P<0.05)$ differences were also found between devices with higher results obtained with Idexx and IRMA compared with Cobas and ABL80. However, in comparison to $\mathrm{PO}_{2}$ results, bias and $95 \%$ confidence interval were smaller and are less likely to cause any changes in clinical decisions. For $\mathrm{pH}$, higher results were obtained with the Idexx machine compared to the three other devices. However, all obtained values were within normal reference ranges at room air $(7.36-7.44)^{1}$ and very small bias and $95 \%$ confidence interval were found. Such small differences are not expected to have any clinical significance. For $\mathrm{PCO}_{2}$ and $\mathrm{pH}$, no interaction between the results obtained from any of the analyzers and health status of the dog was found; differences between analyzers are thus probably the result of systematic error.

In human medicine, several studies have looked into interchangeability of different blood gas analyzers in a clinical setting with variable results. Although some studies concluded that differences in results from POC devices should be deemed acceptable, others identified a clinically relevant variation in $\mathrm{PO}_{2}$ measurements. ${ }^{7-9}$ In the veterinary literature, this is the first report to our knowledge to investigate this clinically relevant 

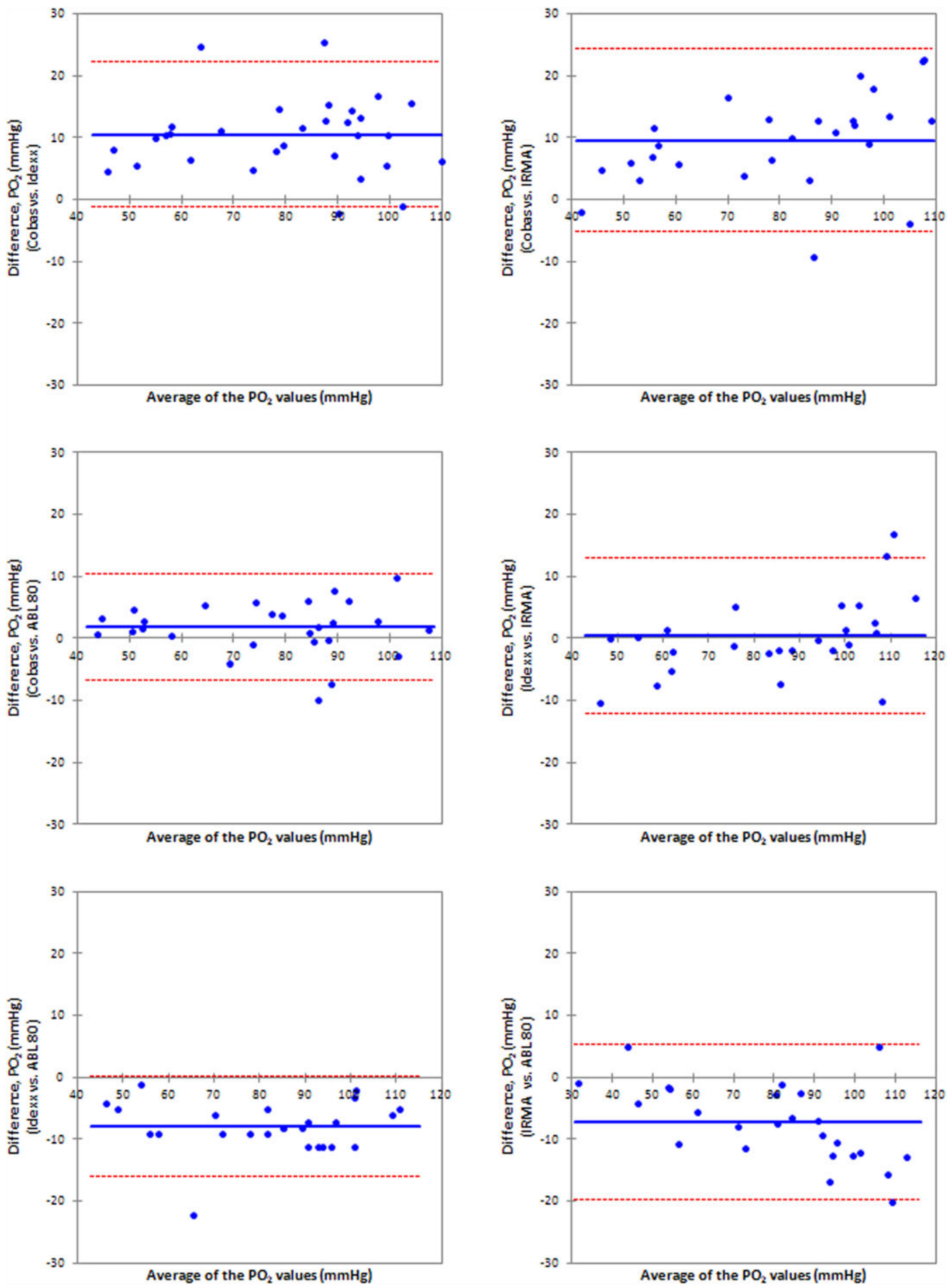

Figure 1: Bland-Altman plots for partial pressure of oxygen results. The $y$-axis represents the difference between results obtained with the two compared analyzers and the $x$-axis represents the average of results obtained by both machines. Biases are represented by a solid line (-) and upper and lower $95 \%$ confidence intervals by dashed lines (---).

question. Our results are in agreement with those published in human medicine concluding that important clinically significant differences exist for $\mathrm{PO}_{2}$ values related to the type of analyzer used. ${ }^{7}$
Regarding the ease-of-use of these machines, no significant differences were found between failure rates of each device. The IRMA device requires manual injection of blood into the cartridge, increasing operator 

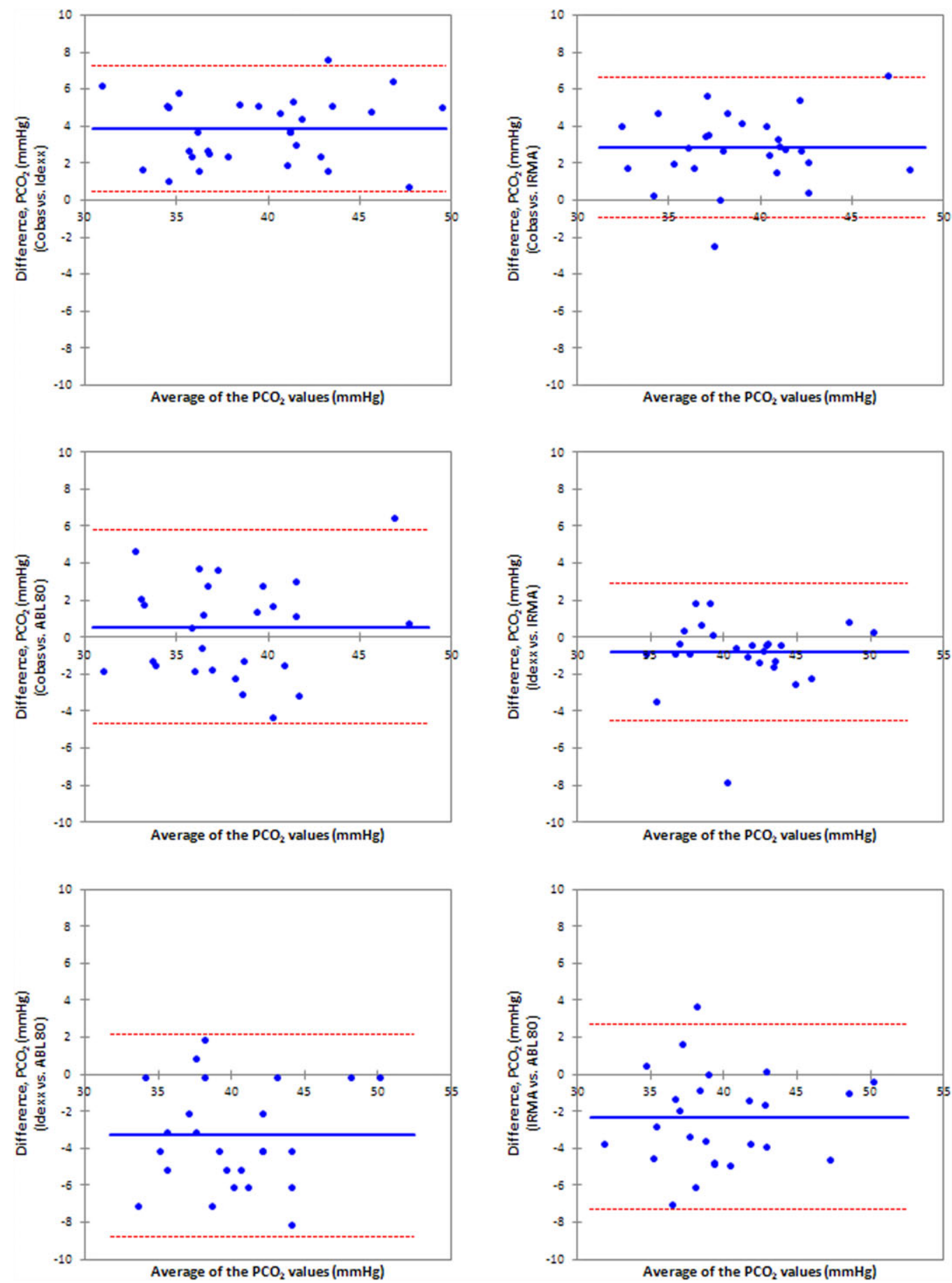

Figure 2: Bland-Altman plots for partial pressure of carbon dioxide results. The $y$-axis represents the difference between results obtained with the two compared analyzers and the $x$-axis represents the average of results obtained by both machines. Biases are represented by a solid line (-) and upper and lower $95 \%$ confidence intervals by dashed lines (---).

dependency, whilst the 3 other analyzers aspirate blood automatically. The Cobas and Idexx devices use an autoaspiration technique while the ABL80 machine requires the operator to manually hold the syringe in place during aspiration.
The first limitation of this study was the low number of dogs included; however, this did not prevent us from highlighting significant differences between devices. A second limitation is the fact that precision was not assessed according to CLSI EP5-A2 guidelines. ${ }^{10} \mathrm{We}$ 

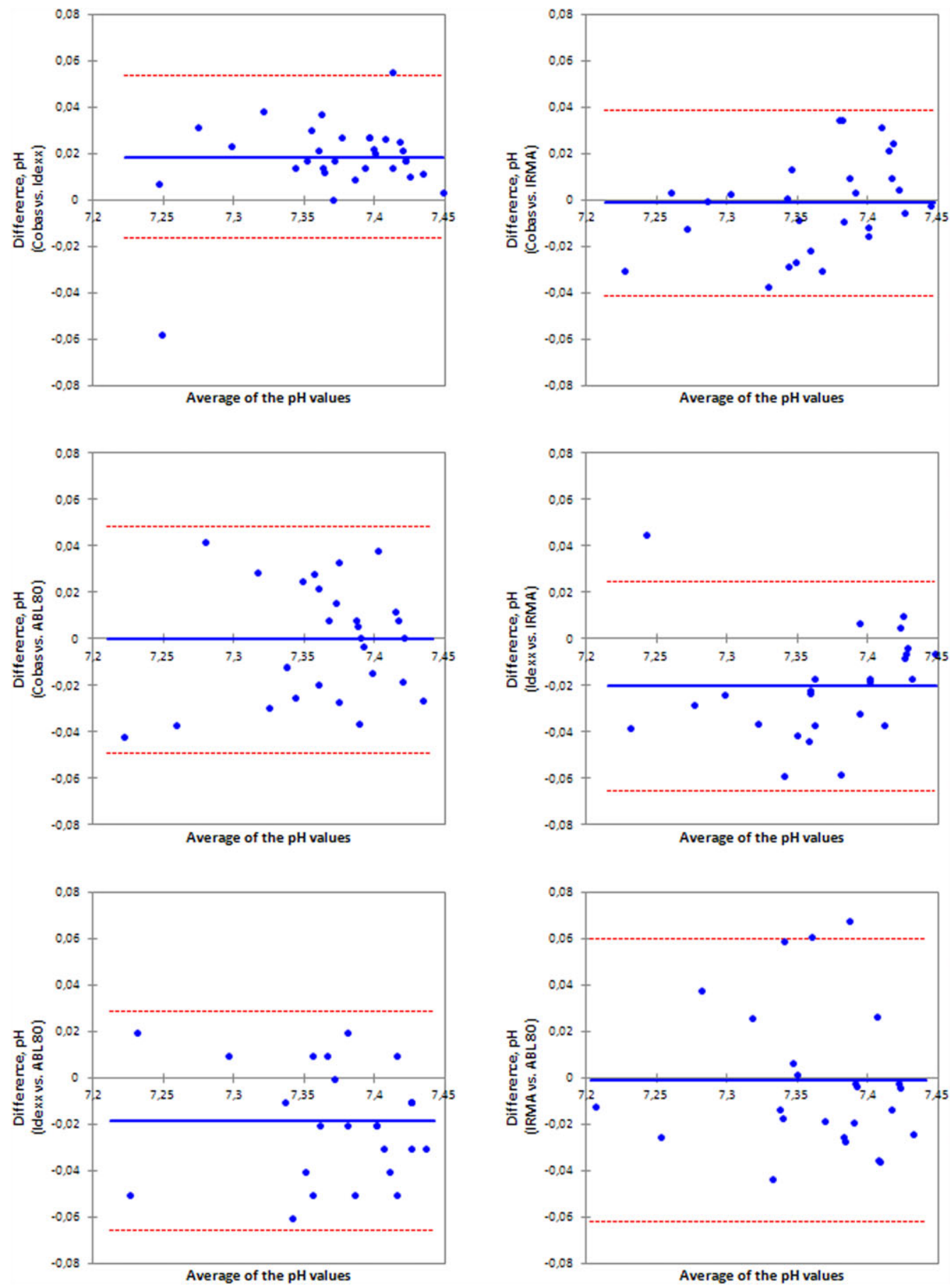

Figure 3: Bland-Altman plots for $\mathrm{pH}$ results. The $y$-axis represents the difference between results obtained with the two compared analyzers and the $x$-axis represents the average of results obtained by both machines. Biases are represented by a solid line ( - ) and upper and lower $95 \%$ confidence intervals by dashed lines (---).

did not find any significant differences between intraanalyzer variance for the 4 devices for any of the variables studied. However, we cannot rule out that this lack of statistical significance may be due to the simplified methodology used in this study.
In conclusion, $\mathrm{PO}_{2}, \mathrm{PCO}_{2}$, and $\mathrm{pH}$ values obtained with different $\mathrm{POC}$ blood gas analyzers differed significantly between devices. For $\mathrm{PO}_{2}$, these differences may be clinically relevant and interfere with diagnosis or clinical interpretation. Therefore, blood gas analysis 
should always be interpreted in the context of the devicespecific reference ranges and serial analyses should be performed on the same device to allow meaningful comparison.

\section{Acknowledgments}

The authors would like to thank Laméris Group SA (Aartselaar, Belgium), Idexx Laboratories (Cergy Pontoise, France), and Radiometer Medical ApS (Bronshoj, Denmark) for providing instrumentation for this study.

\section{Footnote}

a Monovette $2 \mathrm{~mL}$ LH, Sarstedt AG \& Co, Nümbrecht, Germany.

b Linisol $2 \%$ sol., Lidocaine hydrochloride $\mathrm{H}_{2} \mathrm{O}$, B. Braun, Melsungen, Germany.

c Cobas b-123 POC system, Roche Diagnostics, Brussels, Belgium.

d IRMA TruPoint, ITC, Laméris Group SA, Aartselaar, Belgium.

e Idexx VetStat, Idexx Laboratories, Cergy Pontoise, France.

f ABL80 FLEX, Radiometer Medical ApS, Bronshoj, Denmark.

g Mixed model, SAS, version 9.2, SAS Institute Inc, Cary, NC.

h Microsoft Office Excel 2007, Microsoft Corporation, International.

\section{References}

1. McGrotty Y, Brown A. Blood gases, electrolytes and interpretation 1—blood gases. In Pract 2013; 35(2):59-64.
2. Heikkila HP, Lappalainen AK, Day MJ, et al. Clinical, bronchoscopic, histopathologic, diagnostic imaging, and arterial oxygenation findings in West Highland White Terriers with idiopathic pulmonary fibrosis. J Vet Intern Med 2011; 25(3):433439.

3. Hoareau GL, Jourdan G, Mellema M, et al. Evaluation of arterial blood gases and arterial blood pressures in brachycephalic dogs. J Vet Intern Med 2012; 26(4):897-904.

4. Kelmer E, Love LC, Declue AE, et al. Successful treatment of acute respiratory distress syndrome in 2 dogs. Can Vet J 2012; 53(2):167173.

5. Pachtinger G. Monitoring of the emergent small animal patient. Vet Clin North Am Small Anim Pract 2013; 43(4):705720 .

6. Bateman SW. Making sense of blood gas results. Vet Clin North Am Small Anim Pract 2008; 38(3):543-557.

7. Kampelmacher MJ, van Kesteren RG, Winckers EK. Instrumental variability of respiratory blood gases among different blood gas analysers in different laboratories. Eur Respir J 1997; 10(6):13411344.

8. Leino A, Kurvinen K. Interchangeability of blood gas, electrolyte and metabolite results measured with point-of-care, blood gas and core laboratory analyzers. Clin Chem Lab Med 2011; 49(7):11871191.

9. Stadlbauer V, Wallner S, Stojakovic T, et al. Comparison of 3 different multianalyte point-of-care devices during clinical routine on a medical intensive care unit. J Crit Care 2011; 26(4):433 e111.

10. Tholen DW, Kallner A, Kennedy JW, et al. Evaluation of Precision Performance of Quantitative Measurements Methods; Approved Guideline, 2nd ed. Wayne, PA: Clinical and Laboratory Standards Institute; 2004, pp. 7-13. 